\title{
L’industrie en Méditerranée
}

Des produits et des hommes

Industry in the Mediterranean, Products and men

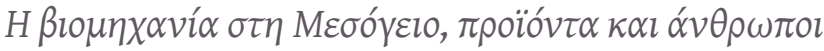

\section{Vincent Gouzi}

\section{OpenEdition \\ Journals}

Édition électronique

URL : https://journals.openedition.org/ceb/10237

DOI : $10.4000 /$ ceb. 10237

ISSN : 2261-4184

\section{Éditeur}

INALCO

Édition imprimée

ISBN : 978-2-85831-294-8

ISSN : 0290-7402

\section{Référence électronique}

Vincent Gouzi, «L'industrie en Méditerranée », Cahiers balkaniques [En ligne], 45 | 2018, mis en ligne le 05 décembre 2018, consulté le 09 juillet 2021. URL : http://journals.openedition.org/ceb/10237 ; DOI : https://doi.org/10.4000/ceb.10237

Ce document a été généré automatiquement le 9 juillet 2021.

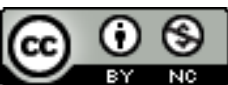

Cahiers balkaniques est mis à disposition selon les termes de la Licence Creative Commons Attribution - Pas d'Utilisation Commerciale 4.0 International. 


\title{
L'industrie en Méditerranée
}

\author{
Des produits et des hommes
}

\author{
Industry in the Mediterranean, Products and men

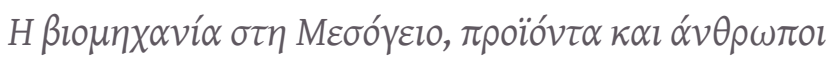

Vincent Gouzi

1 Nous sommes ici, je crois, parce que nous pensons qu'il est intéressant de réfléchir à l'évolution de la place de l'industrie dans nos économies depuis le tournant libéral des années 1970. Et parce qu'il est intéressant de le faire dans l'espace méditerranéen, à la fois parce qu'on ne l'a pas beaucoup fait et parce qu'il est peut-être porteur d'un « modèle » industriel original.

2 On porte encore aujourd'hui un regard très "orienté " sur le processus d'industrialisation. La révolution industrielle qui s'est produite en Angleterre puis en Europe du Nord a été un phénomène si extraordinaire et qui a engendré une telle transformation, qu'il a absorbé pour longtemps l'attention des historiens et des économistes. Les révolutionnaires de 1917 ne pensaient qu'à elle, comme les artisans de la reconstruction de l'Europe en 1945 ou les décolonisateurs du tiers-monde. L'Europe du Sud reste ainsi à l'écart, sa révolution industrielle paraît aux chercheurs tardive, imitative, limitée. Comme le dit Gérard Chastagnaret: «La croissance économique en Méditerranée entrait mal dans le prêt-à-porter conceptuel du développement $»^{1}$. Et pourtant, des industries rentables, en forte croissance et innovantes, ne manquent pas en Méditerranée. La fonderie carthagénoise (dont les techniciens vont nourrir celle du Laurion), la minoterie grecque ou le textile du Pirée, l'étonnant Andréas Hatzikyriakos, créateur des premières cimenteries espagnole, grecque, turque comme les réseaux énergétiques du Sud-Ouest français, en témoignent largement pour le passé. C'est un développement continu que montrent encore récemment la fonderie d'aluminium d'Aspra Spitia en Grèce, les districts industriels italiens, l'espadrille espagnole, en Turquie l'électronique et la construction navale. La Grèce, les Balkans, la Méditerranée 
semblent un champ particulier d'étude où renouveler les problématiques liées à l'industrie :

- Au niveau de la définition et du contenu du secteur secondaire: ainsi du glissement de l'agriculture vers l'agro-industrie et de l'intégration de ces dernières dans un concept de régime, d'écologie diététique, spécifique aux données géographiques de la zone; ainsi de l'externalisation de pans entiers de la production vers des sous-traitants ultra-spécialisés comme les laboratoires pharmaceutiques. Ainsi de l'internationalisation de la recherche et des industries culturelles. Ainsi des processus d'innovation.

- Au niveau de l'apparition de nouveaux rapports de production : l'histoire du travail en Grèce fait apparaître des spécificités, à la fois archaïques et modernes: importance de l'entrepreneur individuel, du travailleur indépendant informaticien, artisan ou chercheur, au sein d'un réseau dense de PME (petites et moyennes entreprises), utilisant des formes de travail et de contrat très souples. Le rôle institutionnel de l'entreprise dans le développement, le rapport entre l'État et l'entreprise, autour du thème de la liberté d'entreprendre, s'y définissent de manière originale.

- Au niveau des espaces de l'industrie grecque : leurs propriétés sont l'occasion de renouveler la réflexion sur les processus de localisation ou d'agglomération spatiale, sur les carrefours ou nœuds d'échange et de production, sur les réseaux. De même les processus complexes à l'œuvre dans les districts industriels italiens, français ou balkaniques, dans les grandes métropoles industrielles.

- Au niveau de l'histoire économique et monétaire : l'actualité de la Grèce en fait un objet d'étude particulièrement riche. L'alimentation en liquidité de l'entreprise industrielle, clé de l'investissement et de l'exportation, est l'occasion d'interroger le rôle des intermédiaires dans la distribution de monnaie.

De nouvelles approches de l'histoire incitent à l'élargissement des études sur l'industrie en tant qu'instrument de transformation sociale :

- En mettant en valeur les temps différents qui composent l'histoire, Fernand Braudel plaçait plutôt l'industrie dans le temps court, celui des changements rapides et des innovations. Il notait, en 1986, dans L'Identité de la France, le changement radical vécu dans les quarante années précédentes par rapport à celui intervenu depuis le néolithique. Certaines permanences demeurent pourtant : l'obsidienne de Milos, le passage de l'Égée (la marine grecque), certains champs culturels (l'orthodoxie, la Méditerranée), la permanence du climat. Il s'interrogeait aussi sur les dates, sur la périodisation de l'histoire, ce que l'on peut utilement faire à propos de la datation de la révolution industrielle dont l'apparition est largement précédée par des activités dites préindustrielles, et qui se décompose elle-même en plusieurs révolutions successives, la dernière, celle de l'informatique.

- En faisant appel au concours des sciences sociales, géographie, sociologie, économie, anthropologie, il invitait à élargir l'approche de l'industrie et à mieux éclairer l'objet: études des processus d'agglomération spatiale, anthropologie du monde du travail, en particulier des nouvelles formes du travail, des réseaux, des migrations et du monde de l'entreprise en s'intéressant aux formes de propriété, à la famille, à l'économie de l'innovation; c'est également une étude de l'économie institutionnelle, qui n'oublie pas les transferts de valeur ajoutée, les comportements de l'homo-economicus, les mécanismes institutionnels de la croissance. Il n'est pas jusqu'à la préhistoire, qui n'éclaire l'industrie, sous l'angle du rapport entre l'homme et l'outil, ou de l'archéologie spatiale.

4 Compte tenu de toutes ces données et réflexions, les interventions ont donc été regroupées en trois parties: le produit (interventions de Vincent Gouzi, 
Christophe Bouneau et Christina Agriantoni), l'entreprise (interventions de Vera Negri Zamagni et Nabil Erouihane), l'emploi (interventions de Léda Papastefanaki, Isil Erdinç et Akis Palaiologos).

- Le produit: la division du travail a une traduction géographique, celle des avantages résultant du climat et de la fertilité des sols, par exemple la diète méditerranéenne, fondée sur des systèmes agroalimentaires localisés ou les fonderies sur l'eau qui s'appuient sur les produits du sous-sol, bauxite, cuivre, argiles industrielles, ou matériaux de construction. Mais pas le charbon et l'acier. Elle a aussi une dimension historique dans laquelle l'innovation joue un rôle déterminant, qui fait émerger des produits nouveaux, de plus en plus immatériels, des produits culturels et des services à l'industrie comme en France.

- Ensuite l'entreprise : il y a évidemment un esprit d'entreprise vigoureux dans ce bassin. Mais il présente des traits que l'on considérait comme archaïques jusqu'à ce que l'essor italien prouve le contraire. La famille méditerranéenne a sans aucun doute subverti le modèle entrepreneurial de la révolution industrielle anglaise et l'on n'a pas fini d'en découvrir les replis. L'artisanat se réinvente un sens moderne, celui que lui donnent les districts italiens, les petits sous-traitants de l'Aérospatiale, utilisateurs de MCN (machines à commande numérique), les informaticiens à domicile. Les grands donneurs d'ordre internationaux actualisent la sous-traitance en confiant le conditionnement, mais aussi une partie de la recherche à des industriels grecs de la pharmacie. L'ouverture des marchés contribue à ces réévaluations.

- L'emploi enfin, la représentation du travail, les conflits professionnels ne peuvent pas ne pas réagir à l'évolution du produit et de l'entreprise ou s'adapter à leurs traits propres. Une société profondément marquée par ses traits culturels méditerranéens (famille, État), s'adapte à l'innovation, s'insère à sa façon dans le jeu de l'offre et de la demande.

Voilà sans doute une vision élargie de l'industrie, un peu moins « économique » et un peu plus anthropologique, un peu moins mécaniste et plus ouverte sur la gestion de l'incertitude. Elle interroge l'évolution du processus industriel: doit-on parler de désindustrialisation des années 1980-1990 ou d'émergence de nouvelles formes industrielles, étroitement liées à des formes d'emploi et de relations professionnelles, dans lesquelles le service nourrit à la fois l'ensemble de la chaîne de production et l'emploi des acteurs?

\section{NOTES}

1. Gérard C hastagnARET, 1997, «L'industrie en Méditerranée: une histoire en construction », Méditerranée, Tome 87, p. 5-12. 
AUTEUR

VINCENT GOUZI

CREE/Inalco/USPC 